\title{
MOTRICIDAD HUMANA Y TEJIDO SOCIAL: ESTRATEGIA PARA COMPRENDER LA DIVERSIDAD Y LA DISCRIMINA- CIÓN EN LOS JÓVENES ESCOLARES
}

\section{HUMAN MOVEMENT AND SOCIAL FABRIC: STRATEGY FOR UNDERSTANDING DIVERSITY AND DISCRIMINATION IN YOUNG STUDENTS}

\author{
Pablo Emilio Bahamón Cerquera*
}

\begin{abstract}
Resumen
Esta investigación se abordó desde un enfoque crítico social, apoyada por los tipos de investigación acción participación, tuvo como objetivo comprender las relaciones de discriminación que vivencian los jóvenes de las instituciones educativas de los sectores vulnerables de la ciudad de Neiva, reconociendo la diversidad de los estilos de vida dentro y fuera de la Institución Educativa. A partir de estas vivencias se determinó junto con los actores, acciones desde la motricidad humana encaminadas a la reconfiguración del tejido social. De esta forma, se indagó a través de testimonios y juicios manifestados por los jóvenes, profesores, directivos y padres de familia sobre la realidad social y académica que viven los jóvenes en el contexto donde interactúa.
\end{abstract}

Palabras clave: Diversidad, discriminación, motricidad humana, tejido social, corporeidad, jóvenes escolares.

\section{Abstract}

The investigation was carried out from a critical-social approach, supported by the types of investigation action-participation, with the purpose of understanding the relationships of discrimination that some young students live in the educative institutes from the vulnerable areas in the city of Neiva, recognizing the diversity of the lifestyles inside and outside the educative institution; since these experiences it was decided together with the actors, actions from the human motor function aimed to the reconstruction of the social fabric. Thus, it was enquired through testimony and opinions given by young people, professors, directors and parents about the social and academic reality that young people live in the context where they interact.

Keywords: Diversity, discrimination, human motor function, social fabric, young people from schools, social network, motor expressions.

\section{Problemática abordada}

En el devenir de la humanidad diferentes seres y formas de vida han habitado el mundo y compartido su andar histórico. En esta perspectiva, se hace referencia a la diferencia de los seres y sus formas de vida, no solo en términos de especie y forma física, sino también en la diversidad que poseen, en sus maneras de ser, estar, sentir, vivir, pensar, soñar y vivenciar la realidad donde interactúan. Pero desde la Antigüedad, se han establecido

Artículo recibido: 26/07/2013 Aprobado: 31/08/2013

* Magíster en Educación, Desarrollo Humano y Valores. Profesor Programa de Educación Física, Universidad Surcolombiana. Email: pabace@gmail.com pabace@gmail.com 
intereses imperantes y en la Edad Media se fortalece el dualismo cartesiano; el cual según Manuel Sergio (2007) considera al ser humano como «un compuesto de dos esencias que se relacionan pero que no se mezclan. Una es la esencia corporal y la otra es la esencia inteligible 0 la razón» (p. 102).

En este sentido, las prácticas, las relaciones, los estilos de vida y las formas de vivir el mundo son controladas por el poder imperante; concepción filosófica que originó la cultura capitalista que busca la explotación irracional del hombre. En este sentido, para Rey y Trigo (2000) «la historia reciente es caracterizada por la vivenciación de una corporeidad instrumentalizada en la cual el cuerpo es considerado, en primer momento, como una herramienta más de producción laboral, un ser que sufre la tensión cuidado-explotación de la producción» (p. 23).

La escuela se ha convertido en una de las estrategias en la reproducción de la cultura dominante, para mantener la hegemonía de su poder. Situación a la cual Mclaren (2005) hace la siguiente alusión:

\section{Es así como la escuela ha sido víctima del dominio del capi- talismo neoliberal, proyectando la idea de ser dominado y manipulado que se percibe sin esencia, sentimientos, emo- ciones, pensamientos, libertad, historia y reflexiones, que debe ser adiestrado e instruido en los elementos necesa- rios para convertirlo en un cuerpo productivo para el capitalismo y el consumo (p. 36).}

Esta postura la complementa Foucault (1976) cuando dice: «la escuela se ha preocupado ampliamente por que los sentidos de vida, relaciones, prácticas y formas de ser, estar, y vivir el mundo, estén encauzado en la disciplina, la obediencia, la docilidad, la fuerza, y el castigo» (p. 72). De este modo, la escuela en un espacio histórico de clasificación, marginación y discriminación, producto de los intereses y las necesidades del paradigma capitalista que busca la cultura dominante y su proyecto globalizador neoliberal.

Pero el modelo capitalista proyectado en la escuela, no solo se conforma con la discriminación, y el rechazo, además pretende sepultar y desaparecer las diferentes formas de ser, de pensar, de estar, de vivir y de sentir sus propias creencias e ideologías, conduciéndolas a un espacio homogeneizador, donde se impone la cultura dominante. Para confirmar lo anterior basta preguntarnos; ¿Por qué siempre un uniforme?, ¿por qué los integrantes de organizaciones políticas son considerados problemáticos?, ¿por qué el chico gay o la chica lesbiana son considerados como pecadores y hasta enfermos? ¿por qué el practicante de los deportes extremos es considerado como vago y drogadicto?, ¿por qué el joven dócil, disciplinado, obediente, religioso, uniformado y productivo, goza con todo el aprecio de la institución?

La situación problemática descrita conllevó a plantear los siguientes interrogantes:

¿Cómo se vivencian las relaciones de discriminación entre los jóvenes, docentes y directivos de las instituciones educativas de la ciudad de Neiva?

¿De qué manera la motricidad humana aporta a la reconfiguración del tejido social en las Instituciones Educativas de la ciudad de Neiva?

El proceso investigativo permitió comprender las relaciones de discriminación que vivencian los jóvenes de las Instituciones Educativas, reconociendo la diversidad de los estilos de vida dentro y fuera de estas instancias. También, permitió determinar acciones que desde la motricidad humana, aportan a la reconfiguración del tejido social, como una contribución a la inclusión, la diferencia y el buen vivir en la escuela.

El referente teórico, se sustentó en los siguientes fundamentos que se describen de manera sintética a continuación.

El tejido social, desde la mirada de Peter Berger, CarIos Beristain, Angélica Castro, la Organización de la Naciones Unidas (ONU) y los aportes del Grupo investigación Molúfode, está asociado a todas las relaciones cotidianas que existen en una comunidad, desarrolladas por grupos y organizaciones que han configurado una subjetividad compartida y legítima. Por eso, la reconfiguración de tejido social en las Instituciones Educativas ubicadas en sectores vulnerables está compuesto por la participación, autonomía, democracia, convivencia, organización y formación socio humanística, las cuales implican mantener una relación directa entre la persona y la sociedad, creando una interacción que permite el fortalecimiento o desgaste de la red de la cual forma parte la persona.

La motricidad humana, es definida por Manuel Sergio (2007) «El movimiento intencional hacia la trascendencia. Movimiento centrípeto y centrífugo de la 
personalización en busca de la trascendencia» (p. 22). Es aquí donde se manifiesta la reflexión y se gesta la crítica, dando paso a la autonomía y la proyección de las diferentes expresiones motrices en los diversos contextos y por distintos sujetos. Por eso, en las expresiones motrices juveniles que tengan en cuenta los principios de la motricidad humana, el cuerpo es una estructura compleja donde emergen el deseo, el placer, la rebeldía, emociones y sentimientos de la forma más variada. Por tal motivo, la corporeidad debe proyectarse de tal manera que contribuya a la formación de personas con saberes integrados, dotadas de habilidades y capacidades para interactuar mental, social y motrizmente con el otro y con el contexto.

Las expresiones motrices, deportivas, artísticas, lúdicas y ecológicas planteadas por (Molúfode, 2009), son potenciadoras de desarrollo y constructoras de tejido social, pues dan sentido al movimiento y abren espacios de interacción en búsqueda de comprender, interpretar y asumir la convivencia ciudadana como espacio de participación y de compromiso para mejorar la calidad de vida.

Comprender la realidad social del mundo juvenil, remite a encontrar situaciones de conflicto, relacionadas a formas, espacios y acciones donde interactúa el joven escolar. Mucho más cuando se tiene claro que la cultura imperante es la buena y nos ha dejado un legado de progreso y de pensamiento único y homogeneizador. Por tanto se cuenta con una identidad sin posibilidades, adulterada, vacía e individualista basada en el consumo, la competición y la violencia.

La motricidad humana, desde su postura filosófica, pretende en los procesos de interacción llevarles a los jóvenes un mensaje y recibir lo suyo y ubicarlos en asombro, en duda y en asimilación, con la intención de compartir lo que realmente se necesita para humanizarlo, ante la situación discriminativa y excluyente. En atención a lo anterior en Colombia se creó la ley 375 del 4 de julio de 1997, con el propósito de dinamizar acciones para el mejoramiento y orientación del mundo juvenil.

Los espacios de interacción escolar en los que transitan los jóvenes, siempre se ven permeados por la dinámica de la tradición, creando un enjambre de discriminación determinada, lo que para Rodríguez (2005) es definido como:
Una conducta, culturalmente fundada, y sistemáticamente y socialmente extendida, de desprecio contra una persona 0 grupo de personas sobre la base de un prejuicio negativo o un estigma relacionado con una desventaja inmerecida, y que tiene por efecto (intencional o no) dañar sus derechos y libertades fundamentales (p. 132).

Por eso la discriminación generalmente termina en agresión, provocada por la falta de respeto a la diferencia que aflora a manera de violencia.

La metodología se abordó mediante el enfoque crítico-social, debido que su interés emancipatorio, permite el libre desenvolvimiento del ser humano como sujetos trascendentales en el contexto donde interactúa. Además se asumió el tipo de investigación acción-participación, que permitió tener una visión global y dialéctica del contexto vivido en las instituciones educativas de la ciudad de Neiva y enfrentar el proceso de manera democrática, donde la realidad comienza a manifestarse a través de la acción reflexiva de los actores. Desde esta perspectiva, se analizaron las percepciones y los juicios manifestados por los jóvenes, profesores, directivos y padres de familia de las Instituciones Educativas Oliverio Lara de la Comuna 6, I.P.C Comuna 8, Gabriel García Márquez de la Comuna 9 y Humberto Tafur de la Comuna 10.

Este proceso inició con el acercamiento a la realidad, la cual permitió los primeros contactos con los grupos discriminados. En segunda instancia, se tuvo como referente las categorías de análisis, que condujeron la aplicación de entrevistas a profundidad, fichas de observación y grupos focales.

Los resultados, se encuentran sustentados en los relatos, voces y actos característicos de los actores que participaron en el proceso investigativo.

\section{Voces y percepciones encarnadas en la motricidad humana y reconfiguración de tejido social en los escolares discriminados}

Desde los primeros pasos en cada una de las instituciones, se logró percibir la problemática abordada, produciéndose una permeabilidad mutua, por eso día tras día, el nivel de empatía entre investigadores y actores era mayor, lo cual permitió un nivel de confianza más elevado que llevaba a ambos a estar más cerca de la realidad problemática. Esto permitió una trascendencia que 
desbordó la capacidad de registro de los instrumentos, donde las respuestas, palabras y gestos, son poseedoras de toda una historia, razón de ser y vida.

Para hacer posible los espacios mencionados de forma pertinente, fue importante comprender en los actores su postura de asumir el cuerpo, el movimiento y el mundo, de forma que les permitiera sentir y vivir la humanización como seres únicos, pero no los únicos que habitan en el mundo. En esta intención, se acudió a la motricidad humana y la corporeidad, como elementos que ofrecen percepciones del yo, el otro, lo otro y el cosmos, modelo que nos lleva a caminar más allá de los paradigmas, cuerpo-mente rico-pobre, hombre-mujer etc., que alejan al mundo de su armónica comprensión.

De esta forma comenzó la vivenciación de las expresiones motrices deportivas, lúdicas y artísticas, en donde los jóvenes se integraban y compartían su diversidad a través del deporte, el juego, la música, el teatro y la danza. También se desarrollaron festivales, en donde los jóvenes impregnados de su intención de trasladarse a nuevas formas de vida, iniciaron a compartir el significado de la motricidad humana y la corporeidad en el mundo, sus vidas y contextos, haciéndose evidente no solo el aprecio teórico por sus conceptos y percepciones, sino en la satisfacción que les proporcionaba el hecho de sentirse más humanos y estar en un mundo en el que ya no se sienten tan extraños.

\section{La colorida e infinita diversidad corporal y la cruel discriminación}

Como raza humana, hemos caminado por el mundo, en muchos momentos sin dejar huella. Lo cierto es que desde aquellos días, hemos pisado el mundo como cuerpos reconstruyéndonos en cada paso, por ello, no es atrevido expresar que no existe una corporeidad igual a otra, haciendo referencia a la corporeidad en palabras de Merlau-Ponty (1956) como «la experiencia de ser cuerpo» (p. 23). Es decir, la corporeidad y universo establecen una relación de significación mutua, situándonos en un avatar constante que nos lleva a una mayor complejidad y nos hace más humanos.

Durante los acercamientos en el proceso investigativo, fue notable como en todas la Instituciones Educativas existían variedad de formas corpóreas, dentro de una amplia diversidad cultural, sexual, religiosa, racial, de clase y de género, entre otras categorías. A partir de sus relaciones y lenguajes fue sentido que existían situaciones de discriminación y de alienación de algunas de esas formas vividas, en donde el vivenciar de la diferencia y su compartir, no dejaba gran espacio a la armonía y el respeto.

Fue por ello que en un primer momento se acudió a los estudiantes, quienes expresaron: *Existe bastante discriminación sobre compañeros que son considerados feos, calvos, de pequeña y alta estatura; a otros por ser flacos o gordos les hacen el "chay», por su aspecto físico. Pero lo peor es que las personas que discriminan no se miran como son*. Uno de los psicorientadores entrevistado frente al tema expresó; " $c u a n d o$ los estudiantes se preocupan por su figura corporal hay al menos dos explicaciones: la primera es el cuidado de su cuerpo derivado de una cultura de la estética. La segunda es la sentida por las niñas, que no son aceptadas por las que se consideran que tienen medidas perfectas*.

Según los actores, la forma de discriminación más evidente y sentida por los estudiantes, se encuentra dimensionada en lo estético, en donde solo son válidos los cuerpos instaurados dentro de la voluptuosidad e hipertrofia, que son exhibido y exigido por el mercado como símbolo de belleza. Juicio compartido por Morris (2000), quien expresa «que el paraíso en la tierra requiere ahora simplemente el aspecto brillante y musculado, venerado en Hollywood, como símbolo de perfección» (p. 28).

\section{La sexualidad una forma de vivenciar la corporeidad}

Con relación a la complejidad humana, Sergio Toro (2005) afirma; «la parte actualiza el todo y el todo actualiza la parte» (p. 17), ante esto cabe mencionar, cómo la sexualidad sobrepasa el gusto por un hombre o una mujer, y que como parte de un todo, es significada por el cúmulo de fenómenos políticos, sociales, culturales, religiosos y económicos, que nos rodean, así como de todas las otras dimensiones que como ser humano complejo nos integran; porque no existen dos corporeidades iguales, lo que implica dos formas iguales de ser sexualidad en este mundo.

Por ello se acudió a las aulas y a jóvenes, quienes referente a la vivenciación de su dimensión sexual, ex- 
presaron; *en la actualidad existen diferencias y discriminación sexual simbolizadas en el machismo; evidenciando humillación en contra de las mujeres, además, existen fuertes acciones de discriminación con los compañer@s que viven el homosexualismo y lesbianismo, a quienes en ocasiones agreden de manera fisica y verbal*. Juicio que comparte los profesores Psicoorientadores, quienes manifestaron que *.existen acciones orientadas a la discriminación sobre el homosexualismo, el lesbianismo y la división entre los educandos de sexo masculino y femenino*.

\section{Sentidos y significados más allá de la piel}

Habitamos un continente plagado de razas diferentes a la de los poderosos del occidente y del norte, en donde nos distinguimos de ese tono blanco, por toda una gama de colores y tonalidades que parece infinita, que representa toda una historia, una cultura, un lenguaje, un sentir, un pensar, un estar, un desear, en suma, una diversidad de formas infinitas de vivenciar el universo. Hoy más que nunca debemos ser conscientes de cómo existen fuerzas imperiales pretendiendo instaurar las razas de occidente y del norte, como las únicas formas de vida posibles, llegando a crear discursos de carácter discriminativo que pretenden hacer sentir a otras razas inferiores. Como víctimas de ello, se encuentran la raza afrodescendiente, mestiza, e indígena.

En diálogo con los estudiantes, de forma muy sentida expresaron, que *existen muchas acciones de discriminación con relación al color de piel, por ejemplo a un compañero, por ser de raza negra, lo irrespetan en todo momento llamándolo «negro»*. Lo mencionado lo confirman los profesores, quienes revelaron la existencia de un alto nivel de discriminación racial, en donde las principales víctimas son afrodescendientes.

\section{Sobrepasando el creer o no creer}

En las últimas décadas, se ha generado una proliferación de religiones, que radicadas en sus creencias y posturas existenciales, han creado diferencias extremas, en las que cada una asume estar más cerca de la verdad. Esta disputa por la verdad existencial, ha conllevado a la discriminación entre los grupos religiosos, a niveles en donde se llega a desconocer a las otras formas de religión como formas válidas y diferentes.
Lo anterior fue confirmado por los estudiantes para quienes *existe y vivencian discriminación por las creencias o preferencias religiosas sobre todo aquellos que viven las perspectivas evangélica y cristiana*. En este sentido, los profesores resaltan: * existe una amplia diversidad religiosa, lo cual se hace evidente a la hora de abordar la clase y en los actos de religiosos*. Los Psicoorientadores manifiestan que *aunque existen diferentes tendencias religiosas, entre los mismos compañeros hablan de estas preferencias, y aún llegan a compartir rituales y actividades*.

En lo hallado, se destaca como algunos pocos actores expresan que no existe discriminación religiosa, pero la gran mayoría revelaron la presencia de actos de discriminación, orientada de forma intensa sobre los integrantes de los grupos evangélicos y cristianos, grupos antagónicos a la religión imperante; la católica.

\section{Ideología: reflexión y crítica comprender ell yo, ell otro y lo otro}

La percepción ideológica orienta nuestro caminar en el mundo y le da características a nuestros pasos y sentido a nuestras formas de ver, vivir y estar. Por eso, dentro de la gama y variedad de formas ideológicas de asumir nuestra vivencia humana, podría decirse que existen dos grandes grupos, en uno de ellos se encuentran las ideologías de características conservadoras y tradicionales, mientras que otro grupo camina sobre las características alternativas, críticas y de deconstrucción. Estas diferentes perspectivas son la base de la diversidad ideológica del mundo.

Uno de los elementos no agradables del andar ideológico del mundo, es su problemática discriminativa radicada en la diferencias posturales frente a la realidad, que incluso han llegado a marcar encuentros históricos de carácter violento. Por este motivo se creyó interesante escudriñar sobre el vivir de la dimensión ideológica en las Instituciones Educativas, con el deseo de explorar sus niveles de tolerancia con relación al tema.

Ante el deseo mencionado los estudiantes, expresan: que *la institución, no acepta revolucionarios, por sus diversas posturas ideológicas*. Además los profesores revelaron: que *la discriminación ideológica no tiene mayor representatividad en las instituciones, pero reconocen que existe confrontación entre el pensamiento izquierda y derecha*. Por su parte, uno de los psico-orientadores expresó: */os 
estudiantes no saben el verdadero significado, ni la importancia de la ideología de izquierda o derecha. Sin embargo existe intolerancia frente a las diferencias ideológicas*.

\section{La academia un modo de sentir, pensar y de actuar en el contexto escolar}

El maestro Freire (2008) en una entrevista, de forma exaltada manifestó que «los procesos educativos y de formación, deben partir de los contextos y la realidad vivenciada por los educandos», lo cual nos invita al apasionamiento por la generación de procesos conectados con los intereses, deseos, sueños, anhelos, posibilidades, potencialidades y dificultades de los educandos, sus familias, y los fenómenos sociales, económicos, culturales, políticos y religiosos entre otros, que hacen parte de su vivencia.

En el proceso se encontró desde la voz de estudiantes que; "existen grandes diferencias por rendimiento académico, debido a que algunos de ellos son los popularmente conocidos como "flojos», mientras otros son estudiosos entonces les dicen; «nerds». También a los estudiantes que han perdido el año, los supuestos inteligentes, los humillan*. Los psico-orientadores, durante las charlas afirmaron: *existen preferencias de los profesores, por aquellos estudiantes que sobresalen en su rendimiento académico y comportamiento, mientras que se crea una barrera con aquellos que no se destacan*.

\section{La reconfiguración de tejido social un reto para mejorar calidad de vida}

Para vigorizar la reconfiguración del tejido social en los estudiantes discriminados y proyectarlos como sujetos trascendentales, se debe fortalecer la participación, la crítica y el compromiso como mecanismos para generar autonomía y un sentido de convivencia que dinamice la organización escolar, de tal manera que los estudiantes tomen conciencia de su propia existencia, de sus capacidades y posibilidades; en sí, el proceso de reconfiguración del tejido social debe construirse a partir de la toma de conciencia acciones, en las cuales se comprometa con la transformación de la sociedad.

Para enriquecer el sentido de reconfiguración de tejido social, los estudiantes y padres de familia en los talleres expresaron que el tejido social; * ${ }^{*}$ s una red o cadena de personas que se unen y luchan para conseguir un objetivo común, en el cual contribuye a mejorar el bienestar, de la comunidad o grupo en particular*. En este sentido la reconfiguración de tejido social busca a partir de las vivencias, experiencias, conocimientos y expectativas realizar un diagnóstico que conduzca a reconstruir esos vínculos de cuidado a partir de procesos de interacción, participación, de tal manera, propenda fortalecer la democracia, la armonía, la paz y la libertad con base en los acuerdos y normas establecidas por la comunidad educativa.

Referente a la participación, los estudiantes en los talleres manifestaron *que es el espacio en el cual se opina, se expresa, se ayuda y donde se aportan de manera individual o grupal ideas sobre un tema o una tarea especifica; aunque a veces hay ataduras y rechazo, se busca la manera de cómo participar, porque en las instituciones educativas los espacios son cerrados, autoritarios y discriminatorios*. Los profesores y los psico-orientadores coincidieron que la participación *es una acción que integra, las personas para tomar democráticamente decisiones indiscriminadamente*; pero los educadores en especial manifiestan que los estudiantes *participan en trabajos grupales, en tareas, en eventos culturales, deportivos, académicos, en varias de estas actividades el educando representa a la institución ${ }^{*}$. En los momentos de observación se detectó que la participación en las actividades es baja, porque los estudiantes en cierta forma se sienten discriminados por su edad y grado de escolaridad.

La autonomía, según los escolares discriminados *es la toma de decisiones, para mostrar habilidades y conocimientos; las decisiones las debe tomar uno mismo, sin la intervención de otros. Sin embargo en la familia y en las instituciones no comparten sus puntos de vista y actos, muchas veces los callan y los regañan; acciones que han conducido a crear el silencio, el conformismo y sobre todo a sentirse discriminados*. Los profesores y psicoorientadores señalan: *pertinente a la autonomía, los estudiantes están muy quedados; por eso algunos se sienten discriminados, pero cuando se brindan oportunidades aflora el liderazgo y la creatividad en el campo artístico y deportivo*. Mientras, en las jornadas de observación, se detectó que los jóvenes cuando participan en la toma decisiones, sus actos los realizan con esfuerzo y se preocupan que las cosas salgan bien, porque lo hacen de manera autónoma y con gusto.

Los estudiantes consideran la democracia como *un derecho de participación para actuar, para ser escucha- 
dos y para elegir los representantes de la comunidad educativa*. Además, afirman que *la democracia en algunas Instituciones Educativas es bastante restringida y discriminativa*. Algunos profesores señalan, *que en muchas ocasiones la democracia se presta para comentarios malintencionados, que lastiman la libre expresión y la autonomía estudiantil*. Los psico-orientadores en términos generales expresan que la democracia en las Instituciones Educativas es de carácter participativa y el ejercicio se proyecta a través del gobierno escolar.

Según los juicios emitidos, es importante que las Instituciones Educativas inicien con la modificación de las prácticas cotidianas de la cultura autoritaria de la democracia, las cuales han venido propiciando discriminación, la diferencia, el individualismo, la violencia y la desigualdad social. Por eso, el nuevo concepto de democracia debe ser orientado como una acción que garantiza el desarrollo armónico de los derechos humanos y la satisfacción de las necesidades, de tal manera que dignifique la calidad de vida de los estudiantes como sujetos transcendentales, pues para Mataruna (2003). «Los seres humanos somos los únicos seres vivos que podemos conscientemente desear y vivir un mundo democrático sin negarlo en una enajenación racional» (p. 58).

Durante los talleres los estudiantes y los padres de familia expresaron que la libertad *es el derecho que tienen todas las personas para actuar y pensar las cosas de manera individual y colectiva, sin discriminación ninguna*. Por eso los estudiantes señalan, que en ocasiones se sienten con mucha libertad, buscan hacer lo que más les gusta y lo que quieren de manera creativa y lúdica. Ellos buscan la manera de actuar, pensar y estar libres. Los profesores dicen que * la libertad viene condicionada en normas que los escolares tienen que cumplir y llevarlas a la práctica, para no ser sancionados y discriminados*. Juicio que complementan los psico-orientadores cuando mencionan que *os estudiantes tiene un manual de convivencia concertado que responde a los intereses y bienestar de todos*.

\section{Las redes sociales un nido de relaciones con el otro}

En los talleres, los estudiantes y padres de familia, en términos generales, manifiestan que las redes sociales *son la unión de varias personas que tienen algunos principios en común y poseen un sentido de identidad que los caracteriza para interactuar permanentemente. Las redes generalmente se encuentran constituidas por: edad cronológica, sexo, género, parentesco, habilidades motrices e ideologías, y se caracterizan por la interacción permanente entre ellos*. Desde esta perspectiva, si la red es positiva y trascendental en lo humano, contribuye significativamente a la configuración de tejido social, teniendo como perspectiva la comprensión humana, el buen vivir y la calidad de vida. Por eso las redes en el presente estudio se abordaron desde la organización, la convivencia y la identidad.

En los talleres, la organización es considerada *como un proceso donde se integra un grupo de personas, para desarrollar una serie de acciones, teniendo como estrategia funciones bien definidas según su nivel de desempeño. Por eso, toda organización debe caracterizarse por la disciplina, la participación, la responsabilidad y la integración, sin discriminación ninguna*. Juicio que complementan los profesores y los psico-orientadores, quienes expresan que la organización es una *estructura de carácter participativo, democrático, solidario que tiene características especificas que la identifican. En las instituciones educativas los jóvenes están organizados en el consejo estudiantil y en algunas subculturas urbanas, que en ciertos modos discriminan*.

En los talleres, estudiantes y padres de familia en términos generales coincidieron que la convivencia es el *acto de compartir o tener una relación respetuosa con la gente que nos rodea, de forma pacífica, sana y agradable; con el fin de tener una buena armonía en el medio donde se interactúa*. Además mencionaron que la convivencia en la instituciones se caracterizan *por el irrespeto, la intolerancia, la diferencia; este comportamiento se genera por la violencia existente en el país*. Pero los estudiantes y padres de familia de la Institución Educativa del barrio Limonar, comentaron que dentro la institución hay buena convivencia debido al compromiso que tienen los profesores, directivos y estudiantes para aplicar el modelo pedagógico establecido en el PEI.

Los profesores y psico-orientadores en términos generales relatan que los niveles de convivencia en las clases son agradables, pero en ocasiones las diferencias de sexo, talla y capacidades físicas y maltrato erosionan lastimando la convivencia*.

En los momentos de observación, se detectó que la convivencia se caracteriza por aspectos positivos y negativos. Dentro de los aspectos positivos se destaca el respeto, la tolerancia y el diálogo, como medio 
fundamental en el cual los estudiantes departen hablando en pequeños grupos. Los aspectos negativos de convivencia se observaron sobre todo en el descanso, en la salida hacia las casas y en algunas clases de Educación Física; en estos espacios los estudiantes se agreden física y verbalmente, mostrando altos niveles de intolerancia y de discriminación.

Munera (2007) expresa: la «identidad viviente no solo comporta una multiplicidad de facetas, pertenencias, y dependencias» (p. 132). Además el misma autora manifiesta que la identidad "contiene multiplicidad y unidad, originalidad y conformidad, unicidad y serialidad: necesita siempre del otro, por reproducción y eventualmente comunicación.» (p. 132).

Por su parte, los estudiantes expresaron, que la *identidad se encuentra reflejada en todas aquellas acciones y características que los diferencian de los demás; por ejemplo la forma de hablar, de vestir, de comportarse; pero en este momento se encuentra condicionada, por lo que dicen las otras personas, y lo que anuncian los medios de comunicación ${ }^{*}$. Mientras, los profesores y los psicoorientadores manifiestan que la identidad en los estudiantes *está representada en defender su institución, ellos lo hacen como verdaderos guerreros, manifestando frases como "somos estudiantes de la institución y la hacemos respetar por encima de todo*. Además coinciden que no hay falta identidad a nivel personal, porque a los educandos no les gusta que se les cuestione a fondo su problemática, su hacer y sus compromisos.

En el momento de construir y/o reconstruir el concepto de identidad, hay que tener como referente los juicios emitidos por los actores, porque ellos en el cruce de información según sus funciones y generaciones expresan sentidos que se pueden complementar para crear y recrear un concepto contextualizado y amplio que permita ligar el sentido de autonomía, de pertenencia y de pertinencia, con los elementos de multiplicidad, de unidad, de originalidad y de comunicación; de tal manera que fluya la empatía, la diversidad y la diferencia como principios fundamentales para trascender a la corporeidad, a la paz y a la armonía con el yo, con el otro y con el cosmos.

\section{Motricidad humana, cuerpo y corporeidad}

La relación entre motricidad humana y la corporeidad, es aplicada a cualquier contexto a través de acciones y vivencias que permiten pensar, sentir, percibir, comunicar y actuar, de tal manera que el ser humano asuma conscientemente la forma de ser y estar en el mundo.

Al consultar a los diferentes actores expresaron que la corporeidad es el cuerpo que piensa, que vive experiencias con el fin de divertirse y emocionarse*, Porque sin duda, la corporeidad permite comprender, anhelar, vivenciar, querer y amar la vida, valorando y respectando las diferencias y la diversidad humana. En palabras de Merleau-Ponty (1956), "esa persona que vive, siente, piensa, hace cosas, se desplaza, que crece, se emociona, se relaciona con otras personas y con el mundo que le rodea».

Para comprender un poco más el sentido de corporeidad, emitido por los integrantes de la comunidad educativa, se indagó sobre el cuerpo vivido y la corporeidad deseada de los escolares.

Al tener como referente estos postulados, los estudiantes en los talleres expresaron que el cuerpo vivido, *son todas aquellas experiencias que tiene el cuerpo y cada movimiento que se realizan desde el momento que nace hasta el día que muere; pues con nuestro cuerpo podemos expresarnos y demostrar fuerza, vigor, rechazo y amor hacia los demás*. Mientras los profesores y los psico-orientadores comentaron: *los escolares son muy despreocupados, ya que les hace falta tener un mayor respeto y cuidado con el cuerpo, la gran mayoría de los alumnos se dejan llevar por el consumismo y la moda*.

Se evidenció que en algunas instituciones, las niñas son las más superficiales y enmarcan la belleza física como lo más importante. Los hombres por otro lado, enmarcan su cuerpo vivido con rudeza, fuerza y configurado con aretes y tatuajes para mostrar su poderío. Hay resaltar que el fortalecimiento y cuidado del cuerpo, se hace con el fin de responder a la moda impuesta por la sociedad de consumo.

Es evidente, que la corporeidad deseada en los escolares, tiene mucha fuerza aquel cuerpo esbelto, sensual, musculado y seductor, porque estos cuerpos parecen ser sanos, enérgicos, diferentes, alejados de las enfermedades. Desde esta perspectiva, se hace referencia al cuerpo objeto, y no configurado a una corporeidad, en la cual el cuerpo se encuentra inmerso en una interacción infinita y compleja, donde se percibe la subjetividad y lo espiritual de manera equilibrada con lo material. 
Expresiones deportivas, lúdicas, artísticas, ecológicas y académicas, que integran a los jóvenes escolares discriminados.

En el proceso de integración y socialización de experiencias se detectó que en la implementación planificada de expresiones motrices deportivas, las relacionadas con el fútbol, fútbol sala, baloncesto y voleibol, los estudiantes manifestaron que son fundamentales para crecer en sus dimensiones corpórea, social, emocional y psicológica, mostrando la pertinencia de la motricidad en la vida y en la esencia del ser humano como posibilidad de superación de sus dificultades y de sus virtudes. Además fortalecen en ellos elementos tan importantes como la democracia, la creatividad, la autonomía, la solidaridad, la conciencia, la reflexión y la participación.

Las expresiones motrices artísticas relacionadas con las danzas folklóricas, los bailes modernos, el dibujo, el canto, la pintura, la elaboración de manillas y la realización de figuras en origami, son acciones que realizan los escolares con el ánimo de recrearse y compartir con sus compañeros. En las actividades observadas se detectó deseos de transcendencia e iniciativas, demostrando interés en ellas, y colaboración en el ejercicio mismo de la planeación, la organización y la ejecución.

Las expresiones motrices de carácter lúdico, como el juego, el baile, la televisión, los videos juegos, el dibujo y el deporte, en los momentos de observación se apreció que sirvieron como guía para la recomposición de la convivencia, la autonomía, la solidaridad, la toma de conciencia y la interacción social, logrando dejar de lado actos discriminatorios. Pues las actividades planeadas y realizadas se constituyeron en acciones importantes para el desarrollo de la corporeidad y para reflejar conductas que sin duda incursionan en el vivir de los jóvenes escolares discriminados.

En las expresiones académicas relacionadas con la escritura, la lectura de textos, la realización de tareas y los estudios sobre informática, se observó que son acciones de aprendizaje que obtiene el estudiante con cierta irregularidad y sentido de compromiso. Por eso, es importante promover expresiones académicas como los clubes de lecto-escritura, de matemáticas, de sociales, grupos de estudio entre otros, para que los estudiantes asuman con responsabilidad el aprendizaje autorregulado, independiente, creativo, crítico y reflexivo, que corresponda a los deseos y expectativas.

\section{Conclusiones para la discusión y la reflexión}

1. Es importante iniciar la ruptura de la instauración imperante que suscribe los cuerpos a solo unas formas posibles. En este sentido es necesario rescatar que lo que creemos bello e interesante, no nace en la exigencia del mercado sino en nuestra propia subjetividad, en aquello que nos parece agradable siendo capaces de desarraigarnos de las posturas capitalistas sobre el cuerpo. Para ello, es necesario iniciar la construcción de nuevas formas de sentir la corporeidad, en donde caminemos hacia una cultura que respete y valore la diversidad.

2. Es necesario reencontrarnos como seres humanos referente a nuestra dimensión sexual, para vencer los estereotipos que culturalmente nos llevan por momentos a ser injustos al negar la sexualidad y la forma de ser de otros, trascendiendo a un accionar que nos desarraigue de la discriminación y nos haga caminar a la comprensión y el respeto del heterosexual, homosexual, bisexual, transexual e intersexual como formas diferentes de ser y sentir sexualmente el mundo.

3. Fue evidente que el nivel de discriminación racial en las Instituciones Educativas de la ciudad es alto, pues la discriminación no solo se refiere al mencionar apodos 0 expresar bromas con relación a ciertas razas, sino que algunas se orientan hacia la inequidad de posibilidades, pasando por la percepción de ser considerados como diferentes por el color de su piel, sobre hacia los estudiantes afrodescendientes.

4. Existe en cierta forma discriminación sobre sus preferencias y expresiones religiosas, sobre todo con los integrantes de los grupos evangélicos y cristianos, los cuales son formas religiosas diferentes a la imperante, que es la religión católica.

5. Los actores exigen espacios de libre expresión ideológica, que los encaminan hacia la libertad, la emancipación y la creación de una vida y un mundo diverso, colorido y tolerante, que les permitan convivir en la armonía, en donde cada ideología y sus formas de pensar y asumir la vida, tengan posibilidad de existencia a través del diálogo, el encuentro y el desencuentro que los acerque a un mejor vivir en el mundo. 
6. Desde las voces de los jóvenes, son los docentes los responsables de la creación de estereotipos sobre los estudiantes que no poseen el mejor rendimiento académico.

7. Se reconoció que la autonomía, la democracia, la participación y la libertad son elementos del tejido social, que las Instituciones Educativas permanentemente cierran los espacios y limitan las condiciones para tomar decisiones; porque la cultura autoritaria ha venido violando los derechos humanos, y fomentando la discriminación.

8. Las Instituciones Educativas para lograr desarrollos académicos, administrativos y comunitarios, deben brindar espacios permanentes de libertad, en donde de forma consciente los estudiantes los asuman como formación personal y colectiva, sin diferencia alguna. Por consiguiente las Instituciones Educativas deben promover la creación de organizaciones, que permitan construir redes que se vayan consolidando y brinden sentido de pertenencia e identidad,

9. Es urgente que la Secretaria de Educación del Municipio de Neiva y las Instituciones Educativas, a partir de procesos de integración dinámica y de trabajo colectivo, establezcan estrategias que surjan de las vivencias, experiencias y conocimientos de la comunidad educativa, para abordar un proyecto de convivencia ciudadana, de tal manera que la comunidad académica lo asuma con sentido de pertenencia.

10. Los actores expresaron que en el cuerpo vivido se manifiesta la integralidad plena del ser humano; pero aún existen apreciaciones, en las cuales el cuerpo es limitado a la dimensión física, biológica y estética que busca la belleza y el fortalecimiento del cuerpo como estereotipo de la sociedad de consumo. Modelo que se proyecta evidentemente en la corporeidad deseada en los escolares, porque toma fuerza aquel cuerpo esbelto, sensual, musculado y seductor, 'porque estos cuerpos parecen ser sanos, enérgicos, diferentes, alejados de las enfermedades.

11. Se detectó que las expresiones motrices deportivas, artísticas y lúdicas en el ámbito escolar son satisfactores del ocio, debido a que responden con todas aquellas acciones que generan placer y que ocasionan en el estudiante un cambio constructi- vo y formativo; por tal motivo, deben asumirse como una expresión humanizadora para que los estudiantes actúen sin diferencia alguna como sujetos trascendentales.

\section{Referencias bibliográficas}

Foucault, Michael. Vigilar y Castigar. Buenos Aires: Siglo XXI, 1976.

Freire, Paulo. Pedagogía. 18 de Agosto de 2008. http:/ /www.youtube.com/watch?v=zwri7pO8UHU (último acceso: 12 de Noviembre de 2012).

Maturana, Humberto. Desarrollo humano y ética para la sustentabilidad. Medellín: Universidad de Antioquia, 2003.

McLaren, Peter. La vida en las escuelas. México: Siglo XXI , 2005.

Merlau-Ponty, M. Fenomenología de la percepción. Barcelona: Península, 1956.

Molúfode. Motricidad humana y construcción de Tejido social en sectores vulnerables. Neiva: Universidad Surcolombiana, 2009.

Morris, D. Doença e cultura na era posmoderna. Lisboa: Instituto Piaget, 2000.

Munera, María. Resignificar el desarrollo. Bogotá: Universidad Nacional de Colombia, 2007.

Rey, Ana, y Eugenia Trigo. «Motricidad... ¿Quién eres?» Apunts, 2000:91-98.

Rodríguez, Jesús. El Cotidiano, Definición y Concepto de la no Discriminación. 01 de Noviembre de 2005. http://www.utim.edu.mx/madig/discriminacion/docs/ Articulo JesusRodriguez.pdf (último acceso: 27 de diciembre de 2012).

Sergio, Manuel. En acción 4. Algunas miradas sobre el cuerpo. Popayán: Universidad del Cauca, 2007.

Sergio, Manuel, y Sergio Toro. «La Motricidad humana, un corte epistemológico de la Educación Física» en-acción 1 Consentido, 2005:102. 